\title{
Heptamethine Cyanine Dyes with Robust C-C Bond at the Central Position of Chromophore
}

\author{
Hyeran Lee, J. Christian Mason, and Samuel Achilefu* \\ Department of Radiology, Washington University, St. Louis, Missouri 63110
}

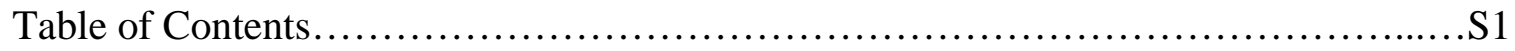

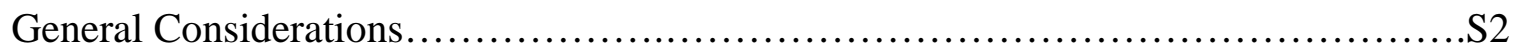

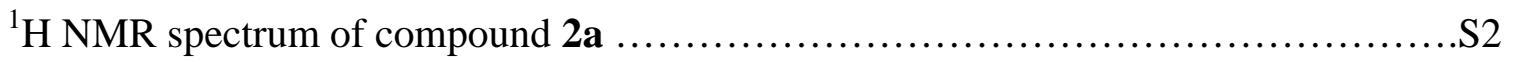

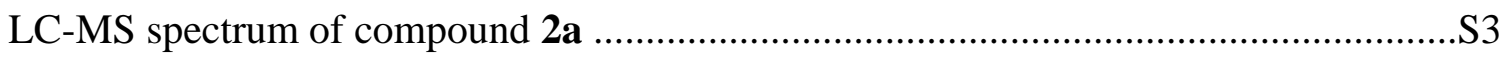

UV-Vis and Emission Spectra of Compound 2a,b ...................................S4

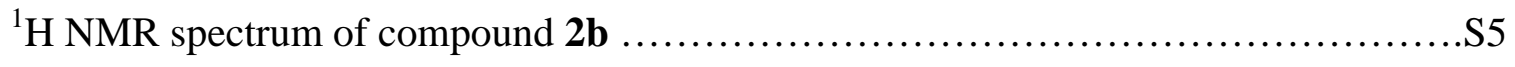

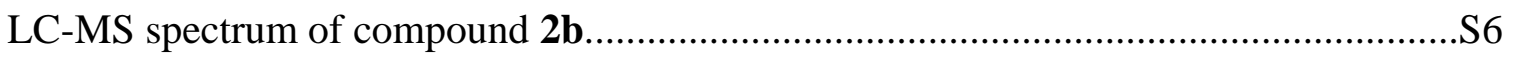

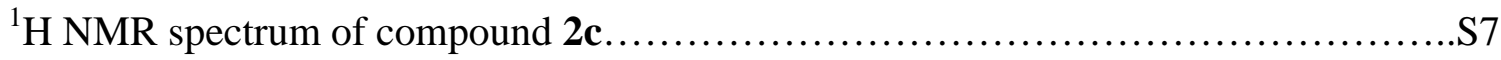

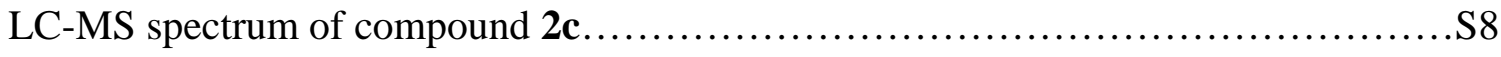

UV-Vis and Emission Spectra of Compound 2c.......................................

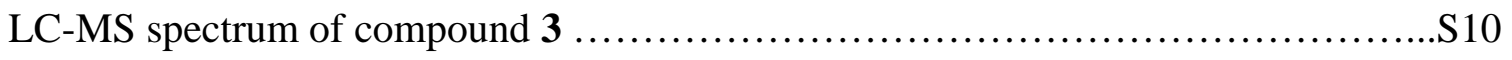

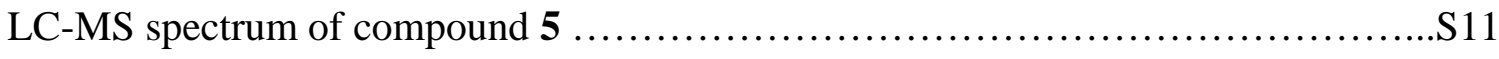


General Considerations. All chemicals were purchased from commercial sources and were used without further purification. ${ }^{1} \mathrm{H}$ NMR data were recorded on a $300 \mathrm{MHz}$ at ambient temperature in DMSO- $d_{6}$ and referenced to tetramethylsilane (TMS) as an internal standard. The absorption and emission spectra were determined using a spectrophotometer and fluorometer, respectively.

${ }^{1} \mathrm{H}$ NMR spectrum of compound 2a

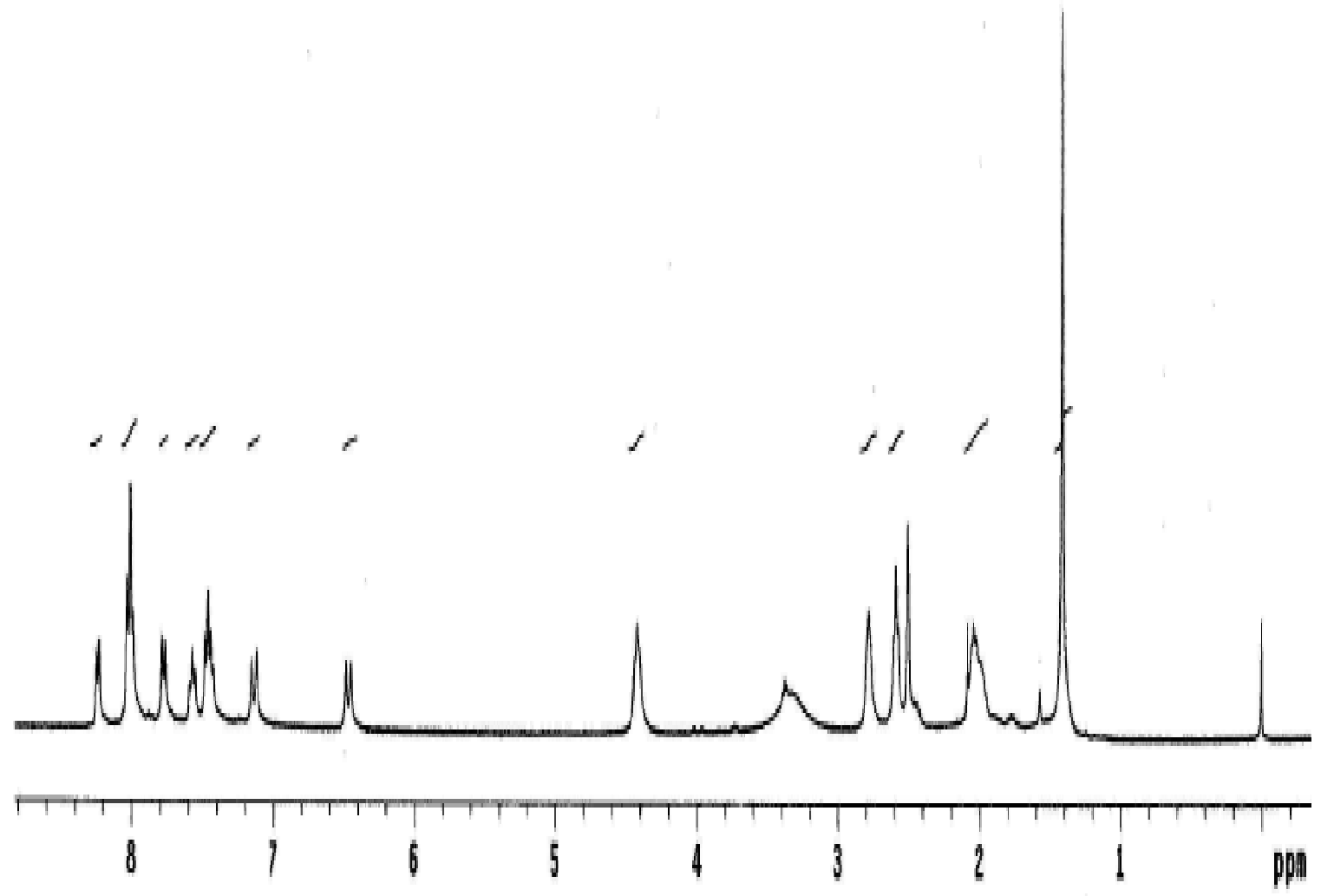




\section{LC-MS spectrum of compound 2a}

$\mathrm{m} \vee$

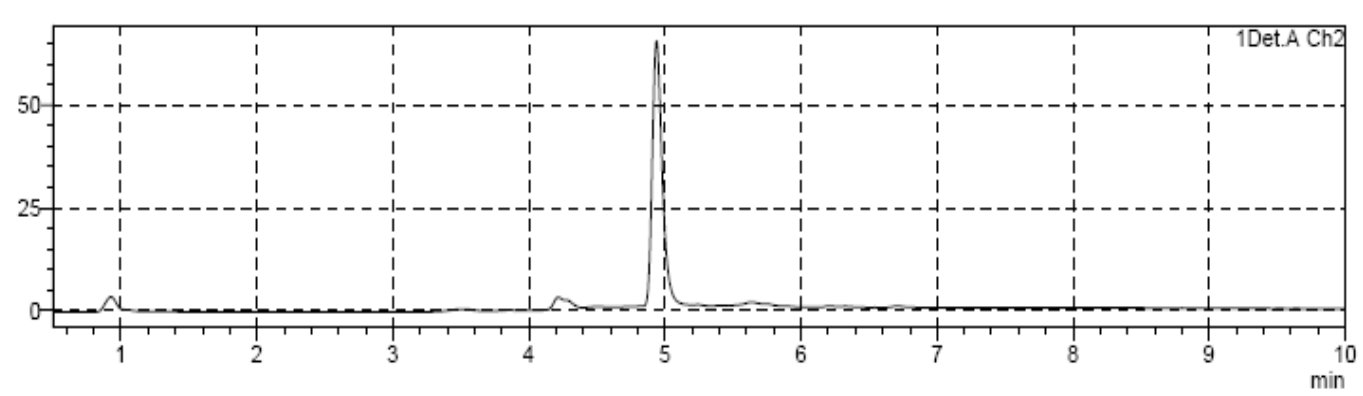

1Det.A Ch2/300nm

MS Spectrum Grapl

Ret.Time:4.925(Scan\#:296)

BG Mode:?

Mass Peaks:1692 Base Peak:226.85(1486700) Polarity:Neg Segmentl - Event1

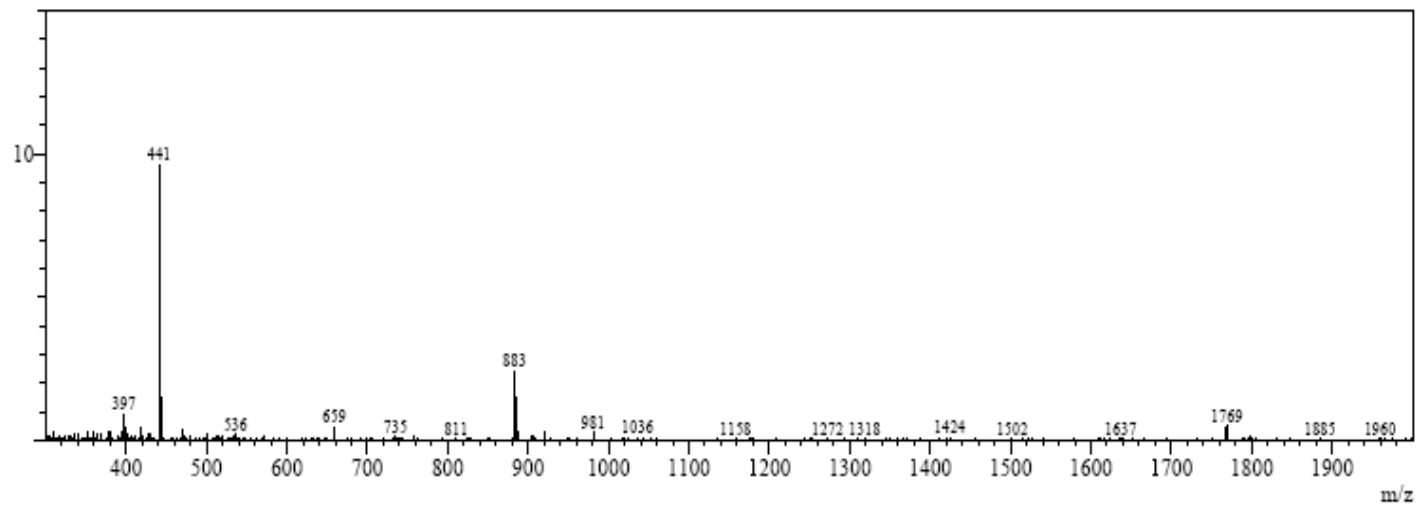


Normalized UV-Vis and Emission Spectra of Compound 2a,b in 20\% aqueous DMSO solution.

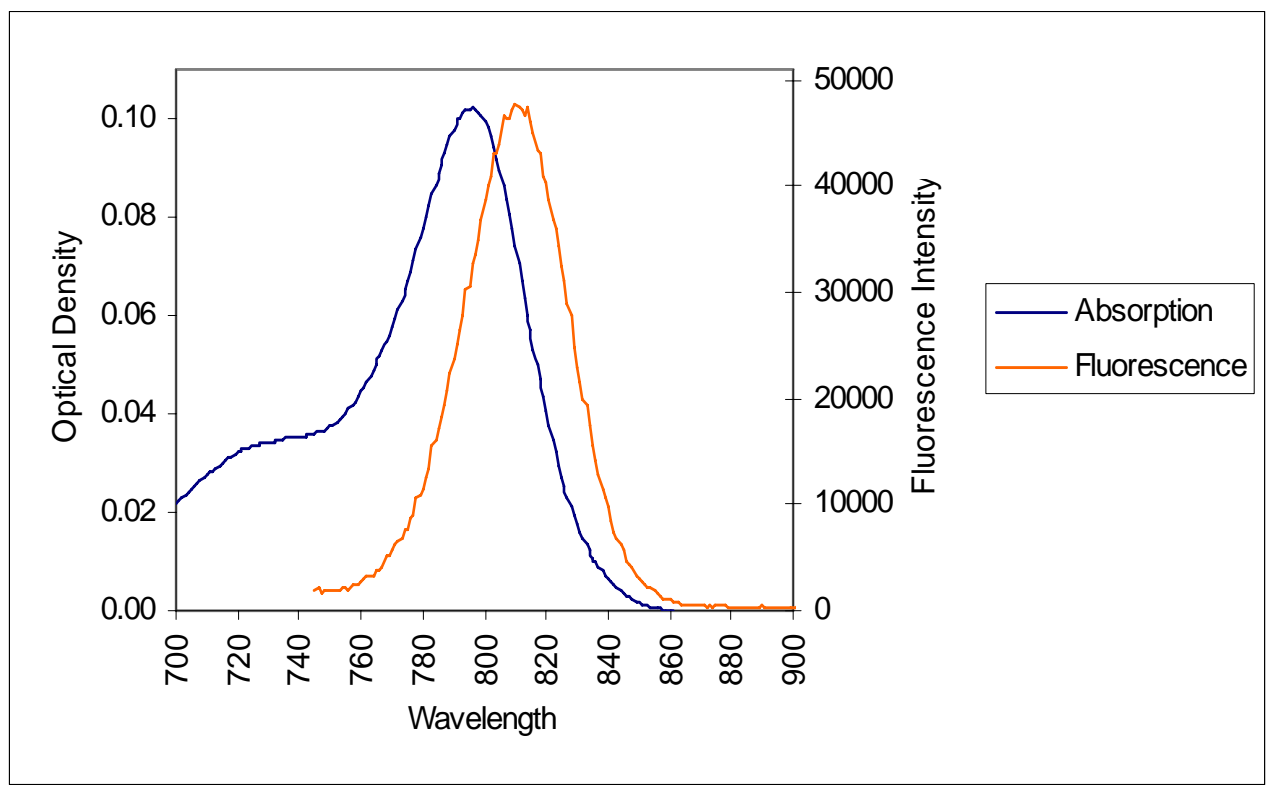


${ }^{1} \mathrm{H}$ NMR spectrum of compound $\mathbf{2 b}$

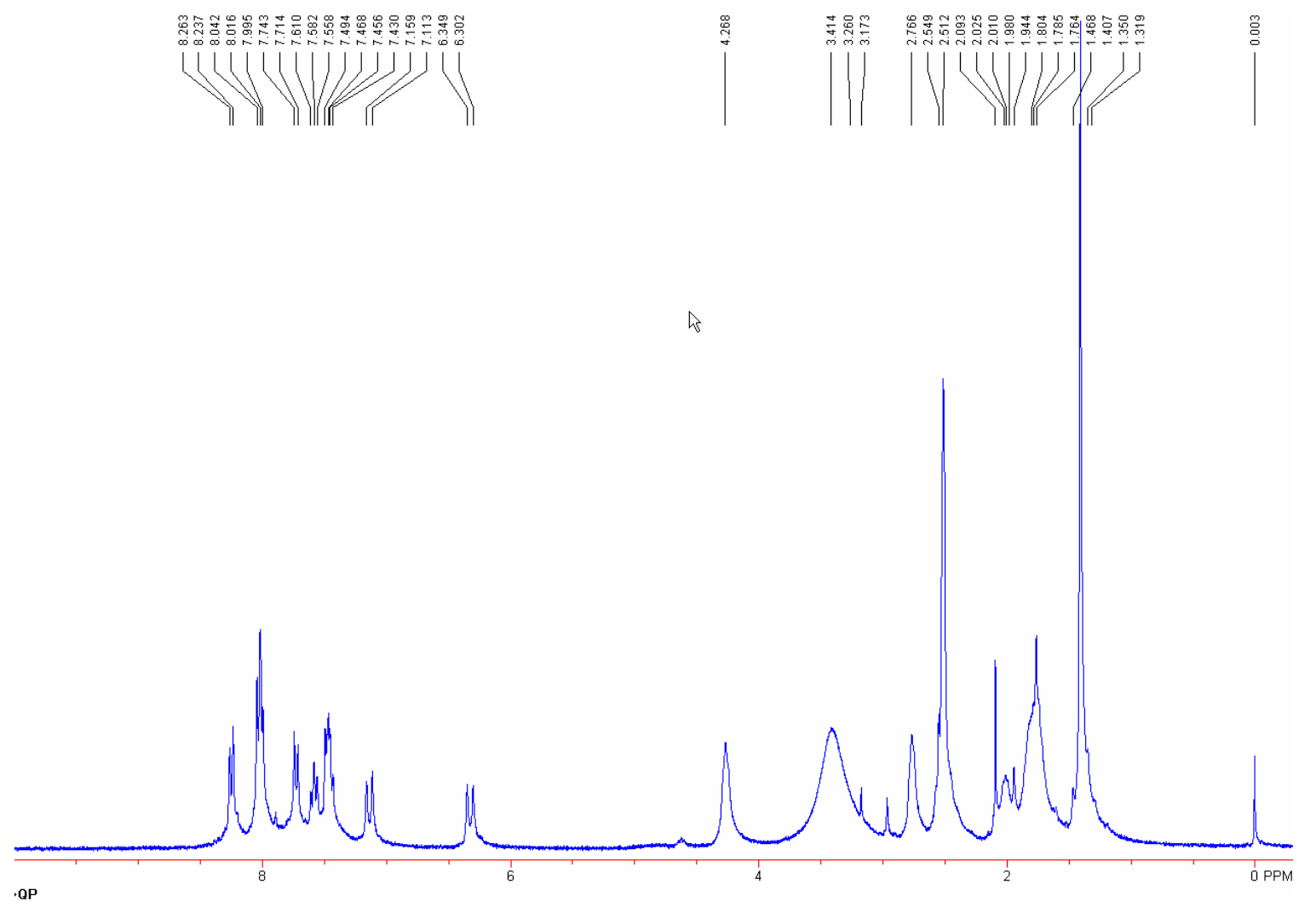




\section{LC-MS spectrum of compound $\mathbf{2 b}$}

$\mathrm{mV}$

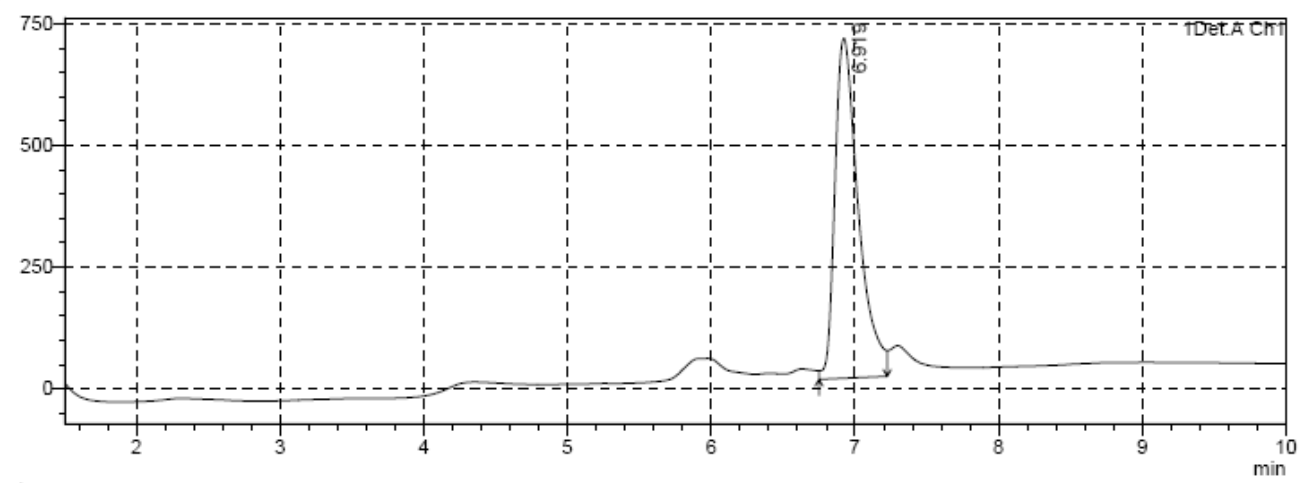

1Det.A Ch1/217nm

MS Spectrum Grapt

Ret.Time: 6.980 (Scan $\#: 433$ )

BG Mode:? 1753 Base Peak:912.90(784002) Polarity:Pos Segmentl - Event]

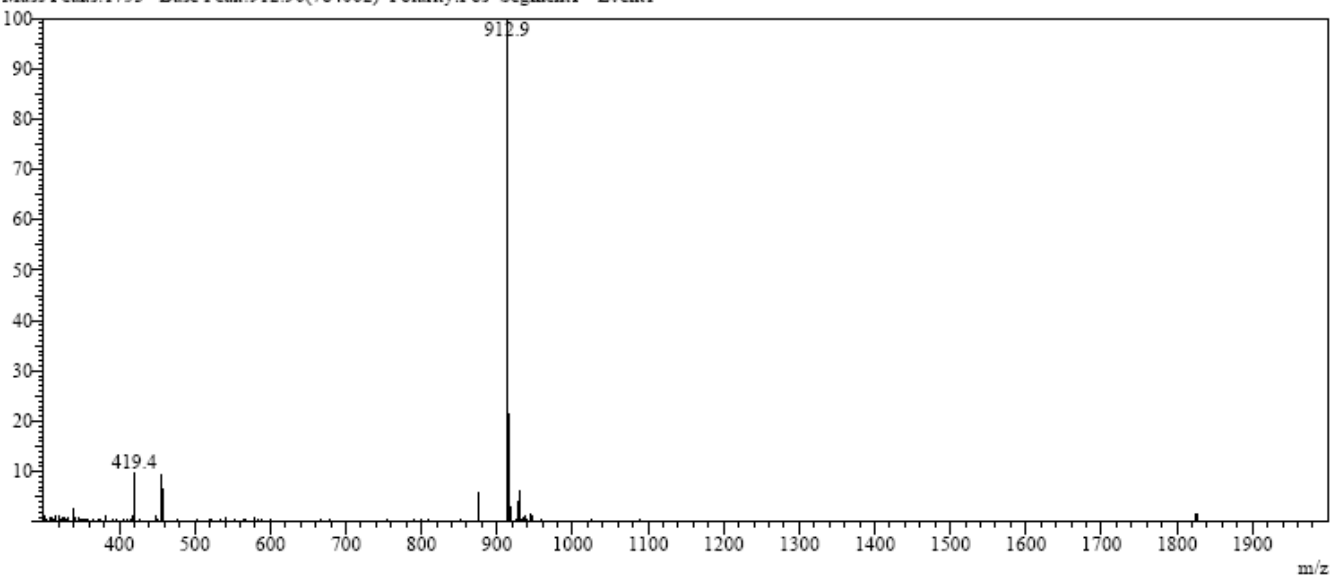


${ }^{1} \mathrm{H}$ NMR spectrum of compound 2c

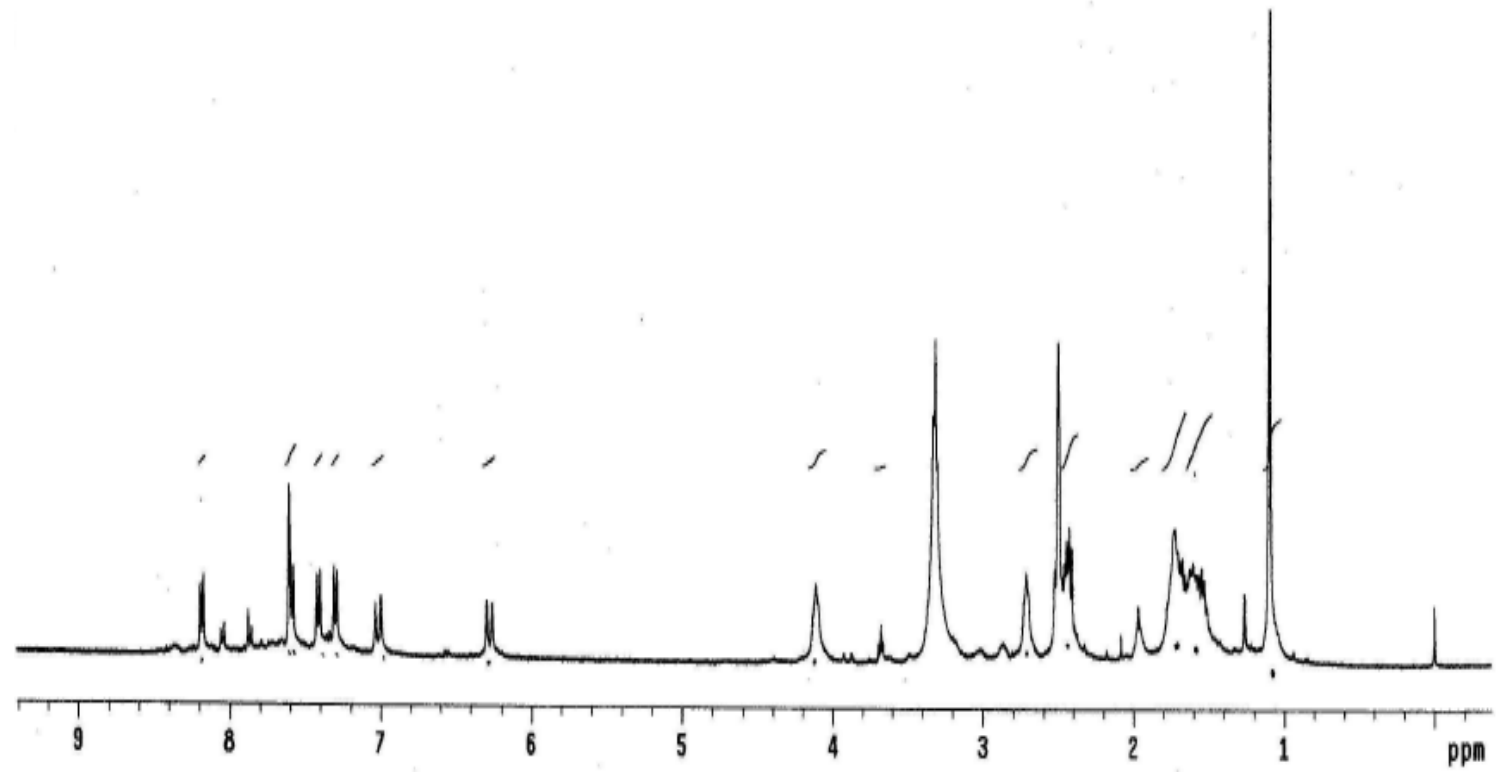




\section{LC-MS spectrum of compound 2c}

mV

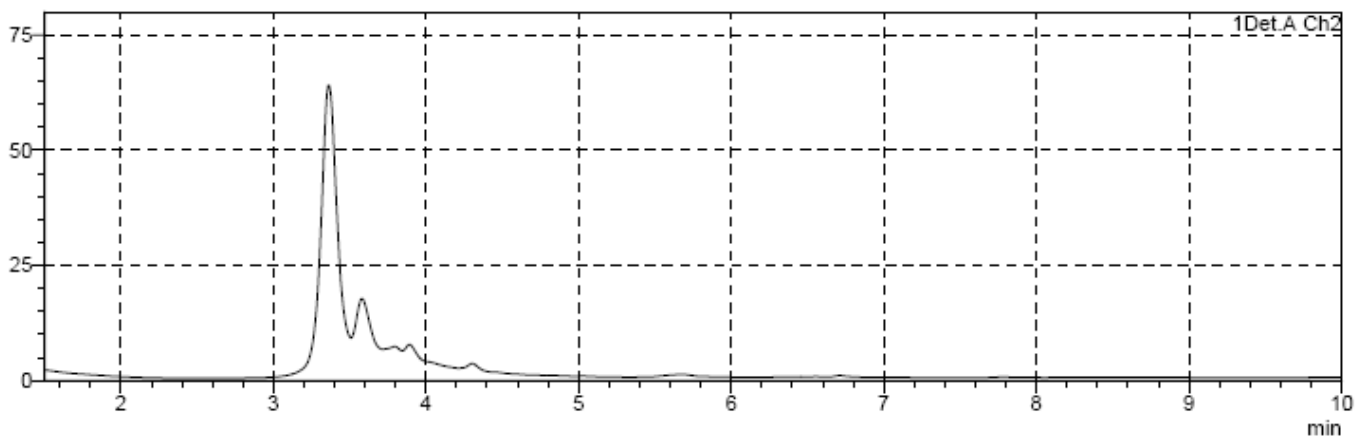

1Det.A Ch2/300nm

MS Spectrum Graph

Ret.Time:3.425(Scan\#:196)

BG Mode:?

Mass Peaks:86 Base Peak:226.85(2690884) Polarity:Neg Segmentl - Event1

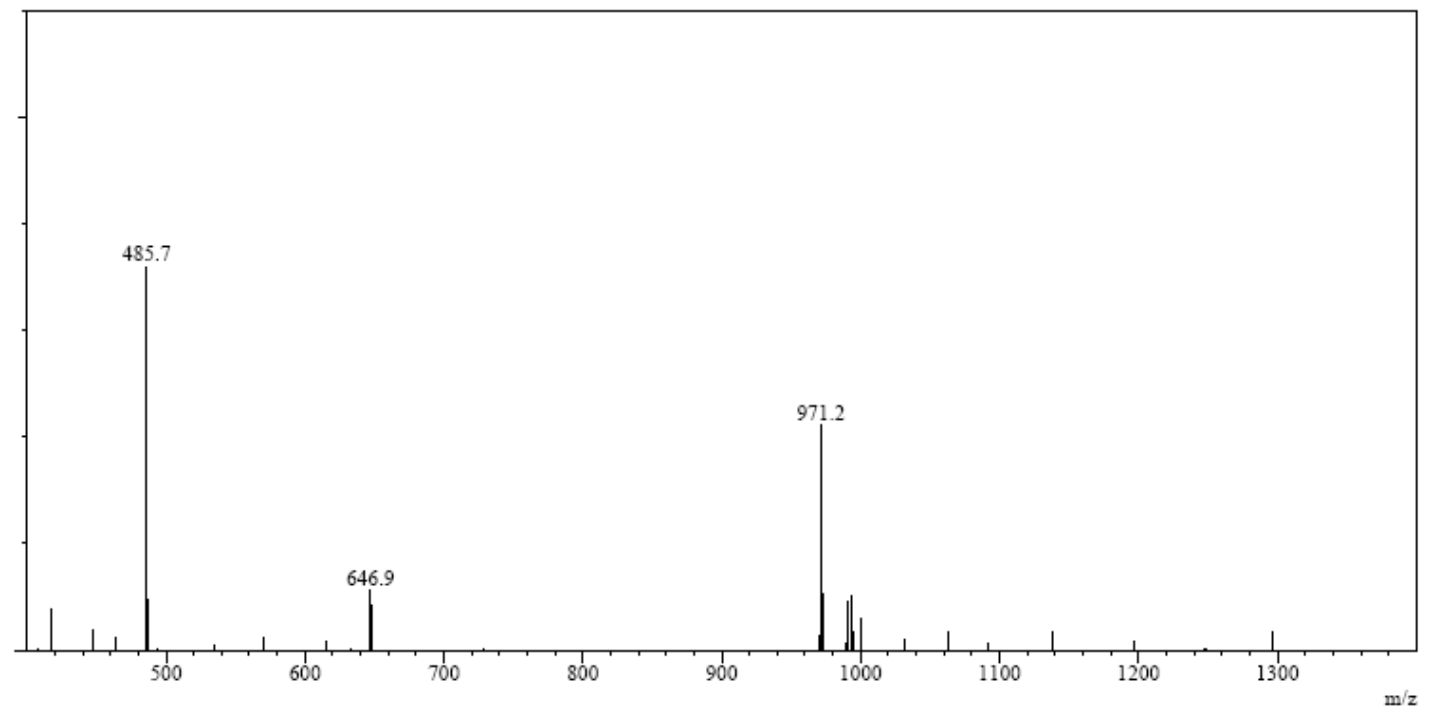


Normalized UV-Vis and Emission Spectra of Compound 2c in 20\% aqueous DMSO solution.

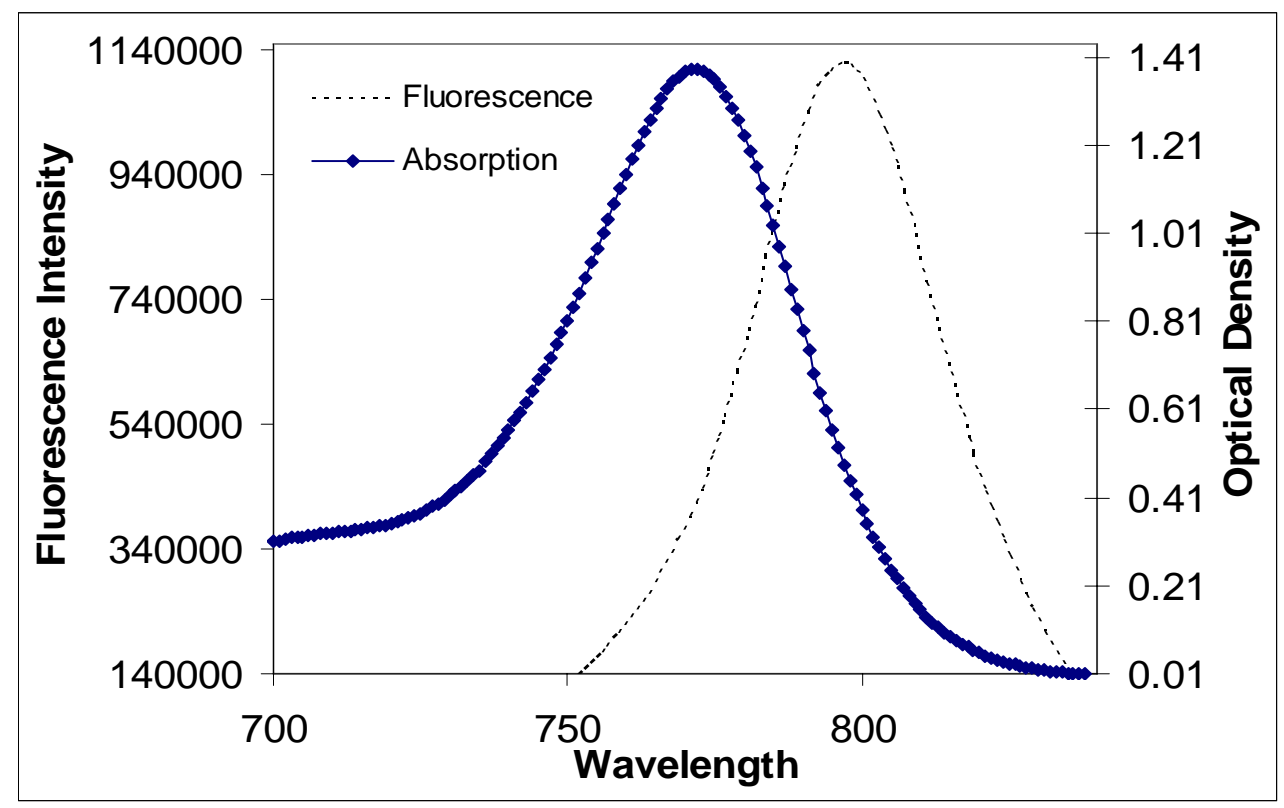


LC-MS spectrum of compound 3.

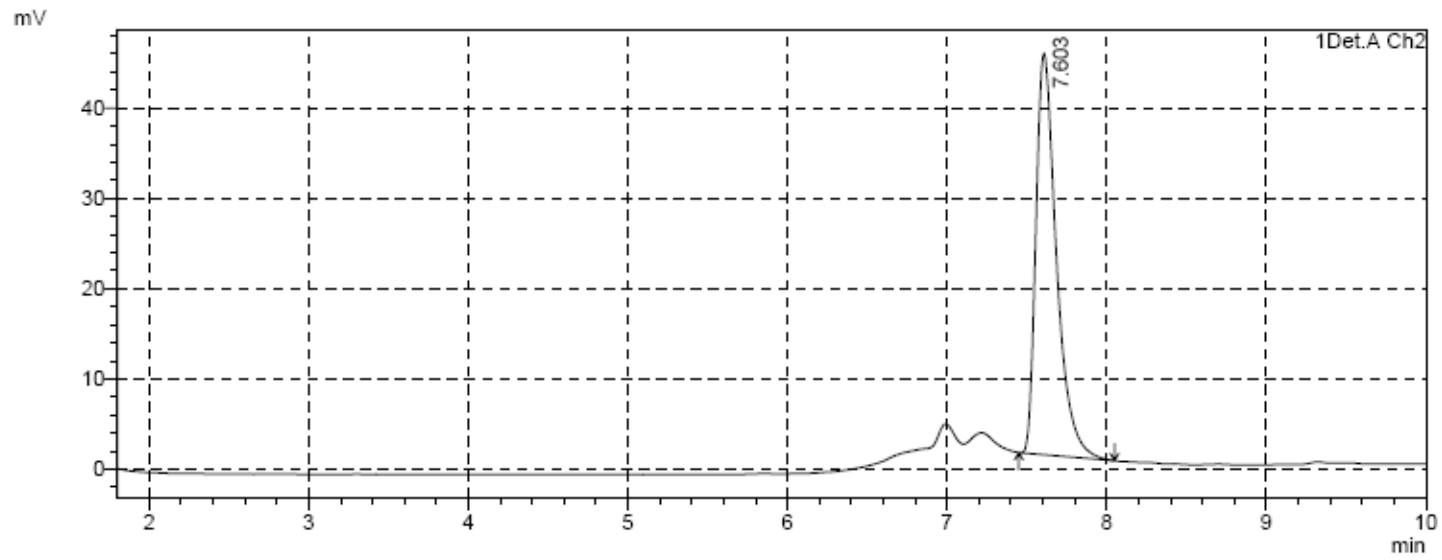

1Det.A Ch2/300nm

MS Spectrum Graph

Ret.Time: $7.670($ Scan\#:479)

BG Mode:?

Mass Peaks:1429 Base Peak:574.80(87752) Polarity:Pos Segmentl - Event1

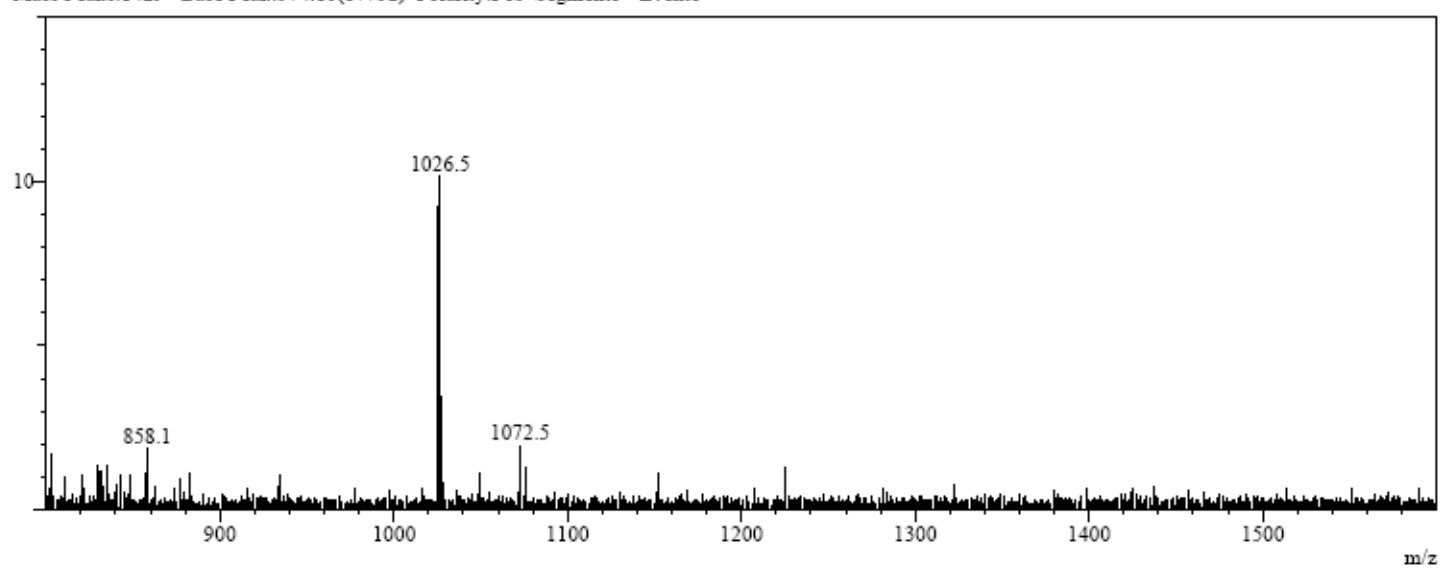


LC-MS spectrum of compound 5.

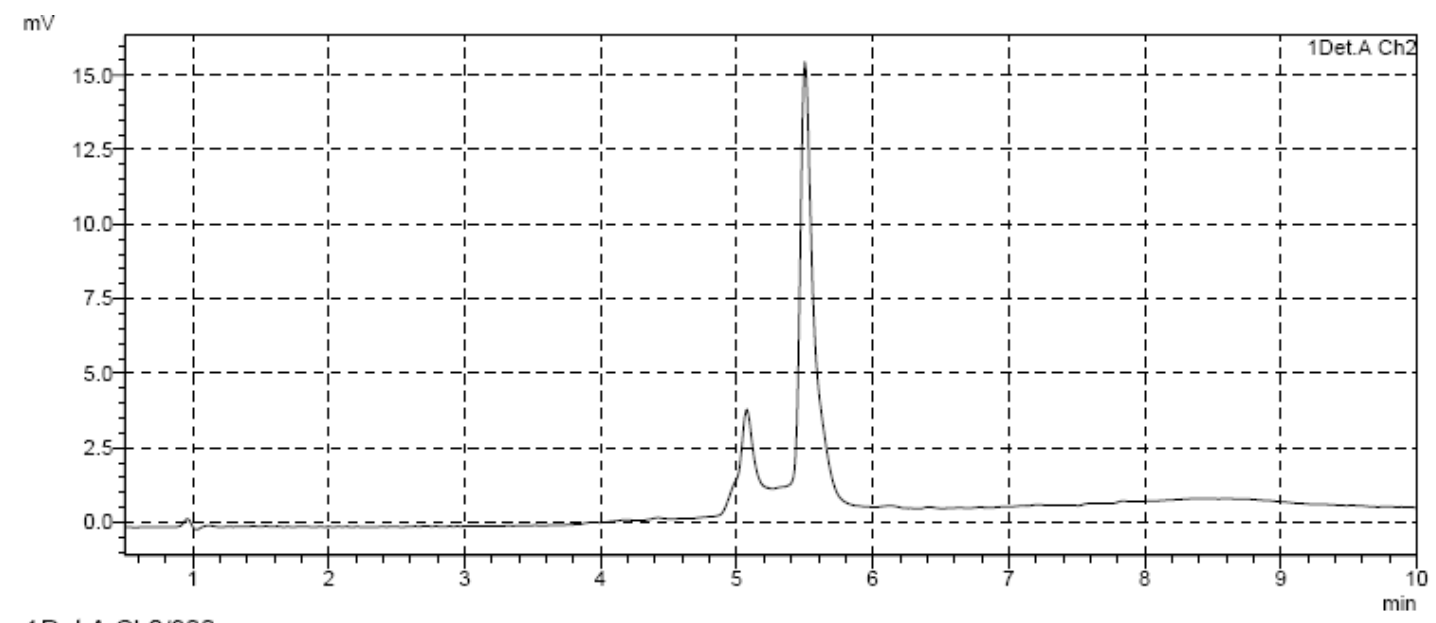

1Det.A Ch2/300nm

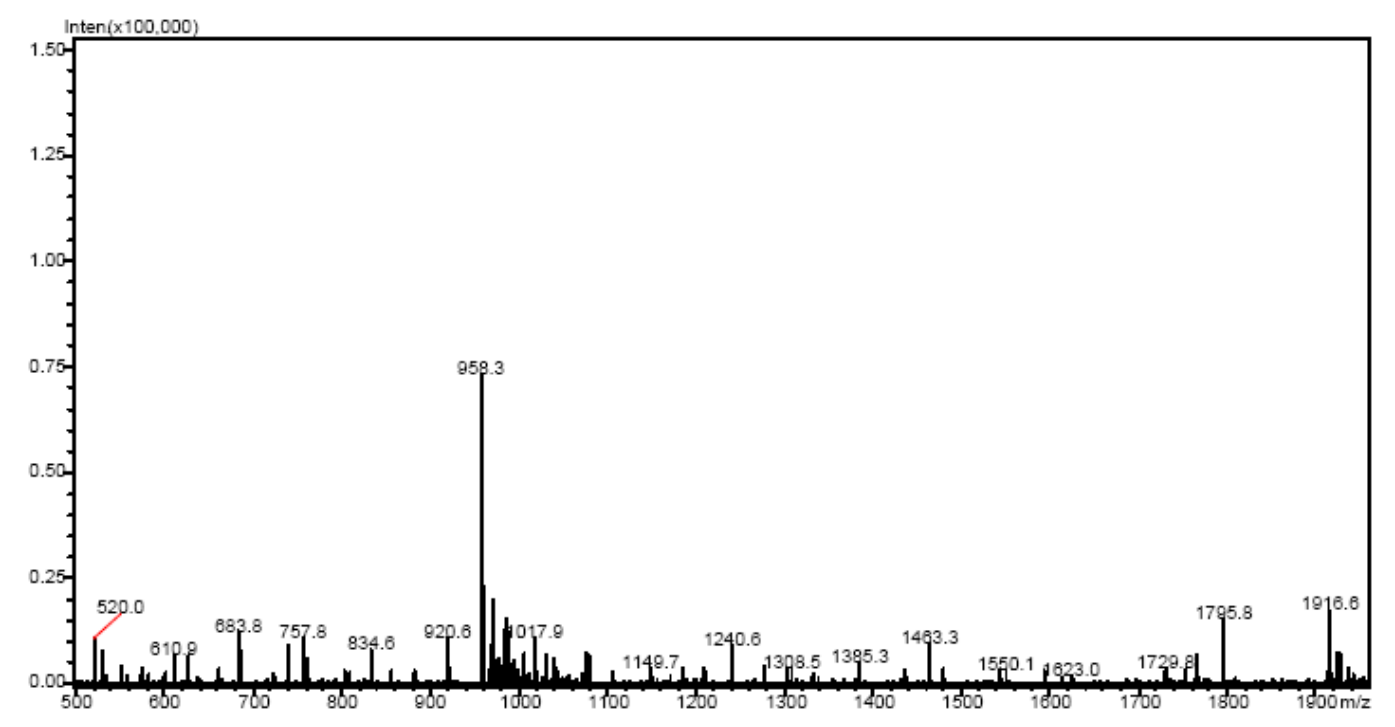

